\title{
Endothelin-1 and Norepinephrine Overflow from Cardiac Sympathetic Nerve Endings in Myocardial Ischemia
}

\author{
Masashi Tawa, ${ }^{1,2}$ Satoshi Yamamoto, ${ }^{1,3}$ Mamoru Ohkita, ${ }^{1}$ and Yasuo Matsumura ${ }^{1}$ \\ ${ }^{1}$ Laboratory of Pathological and Molecular Pharmacology, Osaka University of Pharmaceutical Sciences, Takatsuki, \\ Osaka 569-1094, Japan \\ ${ }^{2}$ Department of Pharmacology, Shiga University of Medical Science, Otsu, Shiga 520-2192, Japan \\ ${ }^{3}$ Department of Pharmacy, Osaka Rosai Hospital, Sakai, Osaka 591-8025, Japan \\ Correspondence should be addressed to Yasuo Matsumura, ymatsumura@gly.oups.ac.jp \\ Received 9 April 2012; Revised 14 May 2012; Accepted 15 May 2012 \\ Academic Editor: Theofilos M. Kolettis
}

Copyright ( $) 2012$ Masashi Tawa et al. This is an open access article distributed under the Creative Commons Attribution License, which permits unrestricted use, distribution, and reproduction in any medium, provided the original work is properly cited.

In protracted myocardial ischemia, sympathetic activation with carrier-mediated excessive norepinephrine (NE) release from its nerve endings due to reversal of NE transporter in an outward direction is a prominent cause of arrhythmias and cardiac dysfunction. Endothelin-1 (ET-1) and its receptors are intimately involved in the regulation of this carrier-mediated NE overflow in protracted myocardial ischemia. The ET-1 system is often complex, sometimes involving opposing actions depending on which receptor subtype is activated, which cells are affected, and whether stimuli are endogenously generated or exogenously applied. Therefore, a detailed understanding of the ET-1 system is important for applying drugs acting on this system in clinical settings for the treatment of ischemic cardiac disease. This article provides a detailed analysis of how the ET-1 system is involved in the regulation of carrier-mediated NE release from sympathetic nerve endings in protracted myocardial ischemia.

\section{Introduction}

Enhancement of cardiac sympathetic nerve activity in myocardial ischemia elicits norepinephrine (NE) release from its nerve endings. Activation of $\beta$-adrenoceptors by NE causes positive inotropic and chronotropic effects which augment oxygen demand. By activating $\alpha$-adrenoceptors, NE also provokes coronary vasoconstriction, which diminishes oxygen supply. The detailed mechanisms by which NE regulates cardiac contractility or coronary vasomotion have been well described in previous reports [1-6]. Both of these actions of $\mathrm{NE}$ are considered to be important factors that exacerbate myocardial ischemia. Indeed, animal and clinical studies have demonstrated that negative modulation of NE release, or blockade of its effects, significantly suppresses postischemic cardiac dysfunction and arrhythmias [7-11]. The mechanisms by which ischemia enhances cardiac sympathetic nerve activity and increases NE overflow have been discussed [7]. In cardiac sympathetic nerve endings in a protracted ischemic condition, free axoplasmic $\mathrm{NE}$ and $\mathrm{H}^{+}$ accumulate massively owing to the lack of a driving force for NE storage by ATP depletion. Increased axoplasmic $\mathrm{H}^{+}$ activates the $\mathrm{Na}^{+} / \mathrm{H}^{+}$exchanger (NHE), which consequently leads to an influx of $\mathrm{Na}^{+}$in exchange for $\mathrm{H}^{+}$. Furthermore, inhibition of $\mathrm{Na}^{+} / \mathrm{K}^{+}$ATPase activity due to ATP depletion results in the accumulation of axoplasmic $\mathrm{Na}^{+}$. This $\mathrm{Na}^{+}$ accumulation triggers excessive axoplasmic $\mathrm{NE}$ release via reversal of the NE transporter (NET) from the intracellular to extracellular space $[6,12]$. This carrier-mediated NE release in protracted myocardial ischemia depends on $\mathrm{Na}^{+}$ entry via NHE activation and is supported by evidence that NHE inhibitors are as effective as NET inhibitors in reducing NE overflow [9]. Although several physiologically active substances (e.g., histamine, adenosine, bradykinin, and angiotensin) contribute to the modulation of this release via respective receptors [9-11], endothelin-1 (ET-1) is also one of them. ET-1 was originally found as a 21 -amino acid vasoconstrictor peptide produced by vascular endothelial cells [13]. Accumulating evidence indicates the crucial role of ET-1 in the pathogenesis and/or development of several 
cardiovascular diseases [14]. It has been reported that an ischemic event results in increases in ET-1 levels [15-18] and its binding sites $[19,20]$. Additionally, blockade of its effects has been shown to act protectively against postischemic cardiac and/or coronary dysfunction both in animal studies $[18,21-29]$ and human clinical trials $[30,31]$. These findings suggest that ET-1 plays an important role in the pathophysiology of myocardial ischemia. In the following paragraphs, we will summarize the interaction between the ET-1 system and NE overflow from cardiac sympathetic nerve endings in protracted myocardial ischemia.

\section{ET-1 System in NE Overflow}

ET-1 is generated from an intermediate form termed big ET1 via proteolytic cleavage by endothelin-converting enzyme (ECE) [13]. The prime sites of ET-1 production in the heart are coronary and endocardial endothelial cells [32-34]. This peptide is well known to have several cardiovascular actions through the activation of its receptors, endothelin type A receptors $\left(\mathrm{ET}_{\mathrm{A}} \mathrm{Rs}\right)$ and type $\mathrm{B}$ receptors $\left(\mathrm{ET}_{\mathrm{B}} \mathrm{Rs}\right)$ [35]. Briefly, both receptor subtypes belong to the seven-transmembrane domain rhodopsin receptor superfamily, and signaling transduction of these receptors is coupled to multiple G-proteins (Gq, Gi/o, and Gs) [35]. These ET-1 receptors are widely distributed in the mammalian heart, such as the coronary vasculature [36], myocardium [37], and cardiac sympathetic nerve terminal [24].

2.1. $E T_{A} R$ s in $N E$ Overflow. Our previous study using the Langendorff technique indicated for the first time that $\mathrm{ET}_{\mathrm{A}}$ Rs modulate carrier-mediated $\mathrm{NE}$ release from cardiac sympathetic nerve endings [23]. In this report, we demonstrated that a selective $\mathrm{ET}_{\mathrm{A}} \mathrm{R}$ antagonist ABT-627 markedly decreased NE overflow in coronary effluent after 40-min global ischemia and reperfusion in rat hearts. A similar result was reported by Isaka and associates [24], who found that $\mathrm{ET}_{\mathrm{A}}$ Rs blockade with $\mathrm{BQ}-123$, a selective $\mathrm{ET}_{\mathrm{A}} \mathrm{R}$ antagonist, significantly attenuated $\mathrm{NE}$ overflow during reperfusion after 20 -min global ischemia in isolated perfused guinea pig hearts. In addition, they provided a definitive demonstration that $\mathrm{ET}_{\mathrm{A}} \mathrm{Rs}$ exist in cardiac sympathetic nerve terminals of guinea pig hearts. These findings suggest that activation of $\mathrm{ET}_{\mathrm{A}} \mathrm{Rs}$ existing in cardiac sympathetic nerve endings modulates carrier-mediated NE release in a stimulatory manner. As support for this proposal, we have reported that 40-min global ischemia-induced NE overflow was more highly observed in isolated perfused hearts of $\mathrm{ET}_{\mathrm{B}} \mathrm{R}$-deficient homozygous $(s l / s l)$ rats than in hearts of wild-type rats, and an exaggerated response to prolonged ischemia in $s l / s l$ rats was abolished by ABT- 627 treatment, indicating that $\mathrm{ET}_{\mathrm{A}} \mathrm{R}$ mediated action is responsible for augmented NE overflow in sl/sl rats [23].

The signaling mechanism of $\mathrm{ET}_{\mathrm{A}} \mathrm{R}$ in stimulatory modulation of carrier-mediated NE release has been suggested. As mentioned above, NHE is an important regulator for carriermediated NE release from sympathetic nerve endings in protracted myocardial ischemia [9]. This transporter activity is well known to be regulated by a variety of G-protein coupled receptor (GPCR) systems [38]. In fact, several researchers have clarified the positive functional coupling of ET-1/ET ${ }_{A} R$ and NHE at a cellular level $[39,40]$. Consistent with this view, in isolated perfused hearts, pharmacological NHE inhibition by 5 -(N-ethyl- $\mathrm{N}$-isopropyl)-amiloride (EIPA) has been demonstrated to decrease excessive NE release induced by $\mathrm{ET}_{\mathrm{A}} \mathrm{Rs}$ activation $[23,24]$. Therefore, it is reasonable to consider that $\mathrm{ET}_{\mathrm{A}} \mathrm{Rs}$ stimulation activates the NHE system at the level of cardiac sympathetic nerves. On the other hand, the second messengers mediating this response remain unclear. Because phospholipase C (PLC)-protein kinase C (PKC) cascade is known to activate the NHE system [38], the stimulatory action of $\mathrm{ET}_{\mathrm{A}} \mathrm{R}$ on carrier-mediated $\mathrm{NE}$ release may result from an increase in PLC and/or PKC activity. However, Horinouchi and colleagues recently demonstrated that there are multiple intracellular signal transduction pathways for $\mathrm{ET}_{\mathrm{A}} \mathrm{R}$ to activate NHE [40]. Briefly, they provided evidence for the existence of an NHE activating pathway mediated through p38 mitogen-activated protein kinase ( $\mathrm{p} 38$ MAPK), not through PLC, in Chinese hamster ovary cells. Unfortunately, at present, we cannot assert which pathway plays a critical role in the positive regulation of NHE activity via $\mathrm{ET}_{\mathrm{A}} \mathrm{R}$ in cardiac sympathetic nerves. It is hoped that the detailed mechanisms of how $\mathrm{ET}_{\mathrm{A}} \mathrm{R}$ stimulates the NHE system and, thus, carrier-mediated NE release in protracted myocardial ischemia will be clarified.

In the above-mentioned previous studies using isolated perfused hearts, NE overflow reflected the severity of cardiac dysfunction after reperfusion. For example, ABT-627 improved left ventricular systolic and diastolic function after myocardial ischemia/reperfusion in rat hearts [23]. Additionally, BQ-123 completely diminished the incidence of ventricular fibrillation after global ischemia in guinea pig hearts [24]. Furthermore, more severe left ventricular dysfunction after myocardial ischemia/reperfusion was observed in $s l / s l$ rats than that in wild-type rats, and this severity in $s l / s l$ rats decreased by treatment with ABT-627 [23]. Basically, $\mathrm{ET}_{\mathrm{A}} \mathrm{R}$-mediated NE overflow is considered to contribute, at least in part, to ischemia/reperfusion-induced cardiac dysfunction.

2.2. $E T_{B} R s$ in $N E$ Overflow. It is still unclear whether or not $\mathrm{ET}_{\mathrm{B}} \mathrm{R}$ directly interacts with carrier-mediated NE release. Our group and another group have confirmed that pharmacological blockade of $\mathrm{ET}_{\mathrm{B}} \mathrm{Rs}$ by A-192621 (selective $\mathrm{ET}_{\mathrm{B}} \mathrm{R}$ antagonist) and $\mathrm{BQ}-788$ (selective $\mathrm{ET}_{\mathrm{B}} \mathrm{R}$ antagonist), respectively, exaggerates $\mathrm{NE}$ overflow induced by protracted global myocardial ischemia in isolated rodent hearts $[23,24]$. As mentioned in Section 2.1, we noted that 40-min global ischemia-induced NE overflow in isolated perfused hearts of $\mathrm{ET}_{\mathrm{B}} \mathrm{R}$-deficient $s l / s l$ rats was more highly observed than in hearts of wild-type rats. These responses to pharmacological blockade and the effects of genetic $\mathrm{ET}_{\mathrm{B}}$ Rs deficiency were almost completely abolished by ABT-627 treatment, indicating the possibility that $\mathrm{ET}_{\mathrm{B}} \mathrm{R}$ itself does not play an important role in carrier-mediated $\mathrm{NE}$ release in ischemic hearts [23]. On the other hand, another group has noted that treatment with the selective $\mathrm{ET}_{\mathrm{B}} \mathrm{R}$ agonist sarafotoxin 
S6c suppresses NE overflow during reperfusion after 20-min global ischemia in isolated perfused guinea pig hearts [24]. More recently, we have demonstrated that $\mathrm{ET}_{\mathrm{B}}$ Rs stimulation resulting from treatment with big ET-1 (see Section 2.5 for more details) suppresses NE overflow induced by 40min global ischemia in isolated perfused rat hearts [41]. In contrast to the above idea, these findings indicate the possibility that $\mathrm{ET}_{\mathrm{B}} \mathrm{R}$ negatively modulates carrier-mediated NE release from cardiac sympathetic nerve endings. The reason for this discrepancy may be due to differences between endogenous and exogenous stimuli. Briefly, the ability of $\mathrm{ET}_{\mathrm{B}}$ Rs stimulation by endogenously generated ET- 1 may be extremely low. As such, the role of $\mathrm{ET}_{\mathrm{B}} \mathrm{R}$ on carrier-mediated $\mathrm{NE}$ release from cardiac sympathetic nerve endings during protracted ischemia is not completely clear and further study is required.

Although it is unclear whether or not there is a direct contribution of $\mathrm{ET}_{\mathrm{B}} \mathrm{R}$ to carrier-mediated $\mathrm{NE}$ release during myocardial ischemia, the amount of NE released from ischemic hearts has reflected cardiac dysfunction in studies introduced in this section. Briefly, treatment with A-192621 worsened left ventricular (both systolic and diastolic) function during reperfusion after 40-min global ischemia, and this detrimental effect of A-192621 was completely abolished by concomitant treatment with ABT-627 in rat hearts [23]. In addition, S6c prevented reperfusion arrhythmias (both incidence and duration of ventricular fibrillation) in guinea pig hearts [24]. On the other hand, BQ-788 did not alter the duration of ventricular fibrillation after 20-min global ischemia in guinea pig hearts [24].

2.3. Endogenously Generated ET-1 in NE Overflow. As described above, we have demonstrated that treatment with ABT-627 with or without the combination of A-192621 markedly suppressed NE overflow induced by protracted global myocardial ischemia to the same level in isolated perfused rat hearts using the Langendorff technique [23]. Isaka et al. also showed that the nonselective $\mathrm{ET}_{\mathrm{A}} \mathrm{R} / \mathrm{ET}_{\mathrm{B}} \mathrm{R}$ antagonist PD-142893 significantly attenuated carrier-mediated NE release during 20-min global ischemia in isolated perfused guinea pig hearts [24]. These findings suggest that endogenous ET- 1 contributes to carrier-mediated NE release by exclusively stimulating $\mathrm{ET}_{\mathrm{A}} \mathrm{Rs}$ in ischemic hearts. This proposal was supported by our recent study which investigated the effects of ECE inhibition [18]. In this study using Langendorff perfused rat hearts, we observed that the selective ECE inhibitor SM-19712 suppressed excessive NE release from sympathetic nerve endings during 40-min global ischemia.

In the previous study, we showed that cardiac ET-1 levels were reduced during ischemia in rat hearts [18]. However, this does not mean that ET-1-induced action is diminished during myocardial ischemia. This is because ET-1 levels in coronary effluent from postischemic hearts were slightly, but not significantly, increased in our subsequent research (unpublished data). In fact, the magnitude of increase in ET-1 release observed during myocardial ischemia has been well documented $[15,16]$. Although it is unclear whether or not ET-1 synthesis is increased in ischemic hearts from our research results, there is almost no doubt that the release of this peptide is increased. Released ET-1 during protracted myocardial ischemia may play an important role in carrier-mediated NE release via the activation of $\mathrm{ET}_{\mathrm{A}} \mathrm{Rs}$. Unfortunately, we did not check the effect of SM-19712 on ET-1 levels in coronary effluent from postischemic hearts. However, we have confirmed that cardiac ET-1 levels are not different before and immediately after prolonged ischemia in the case of SM-19712 treatment [18], suggesting the possibility that ET-1 release during myocardial ischemia is reduced by ECE inhibition. Taken together, endogenous ET-1 positively regulates protracted ischemia-induced NE overflow via the activation of $\mathrm{ET}_{\mathrm{A}} \mathrm{Rs}$, although it is unclear whether or not ET-1 is newly synthesized during ischemia.

Regarding cardiac function, PD-142893 and SM-19712 respectively shortened the duration of ventricular fibrillation [24] and improved left ventricular function [18] during reperfusion after myocardial ischemia relative to NE overflow. Therefore, endogenously generated ET-1 seems to be able to promote postischemic NE overflow, and this contributes, at least in part, to subsequent cardiac dysfunction.

\subsection{Exogenously Applied ET-1 in NE Overflow. Exogenously} applied ET- 1 induces the same effects as endogenous ET-1 on carrier-mediated NE release from cardiac sympathetic nerve endings in ischemic hearts. In our study using isolated perfused rat hearts, exogenous ET-1 $(0.03$ and $0.1 \mathrm{nM})$ caused a marked and dose-dependent increase in NE overflow induced by 40 -min global myocardial ischemia. Moreover, this action of exogenous ET-1 (0.1 nM) on NE overflow was completely suppressed by treatment with ABT-627 or EIPA [23]. In accordance with our findings, Isaka and associates also obtained results indicating that carrier-mediated NE release induced by 20 -min global ischemia was augmented by exogenous ET-1 (0.1 and $1 \mathrm{nM}$ ) in a dose-dependent manner and this augmentation was counteracted with the combination of EIPA in isolated perfused guinea pig hearts [24]. Collectively, it is suggested that exogenously applied ET1 positively regulates carrier-mediated NE release, possibly activating the NHE system through $\mathrm{ET}_{\mathrm{A}}$ Rs stimulation in protracted myocardial ischemia.

Exogenously applied ET-1 worsened cardiac dysfunction during reperfusion after global ischemia in association with the amount of NE released from ischemic hearts. In our study using isolated perfused rat hearts, exogenous ET-1 produced severe left ventricular systolic and diastolic dysfunction after ischemia/reperfusion in a dose-dependent manner, but this response was markedly suppressed in the presence of ABT-627 or EIPA [23]. Also, in the report by Isaka et al., exogenously applied ET-1 prolonged the duration of ventricular fibrillation in reperfusion in guinea pig hearts. Furthermore, ET-1-induced ventricular fibrillation was completely diminished by the combination of EIPA [24].

2.5. Exogenously Applied Big ET-1 in NE Overflow. It is well known that big ET-1 itself has no physiological effects independent of its conversion into ET-1 [42, 43]. Therefore, exogenously applied big ET-1 is thought to have qualitatively 
the same biological activities as ET-1. In our recent study using isolated perfused rat hearts, however, exogenous big ET-1 (0.1, 0.3 and $1 \mathrm{nM})$ did not increase NE overflow induced by 40-min global myocardial ischemia as opposed to the case of exogenously applied ET-1, in spite of the fact that ET-1 levels in coronary effluent from the heart exposed to protracted ischemia were dose-dependently increased by exogenous big ET-1 application [41]. To be more precise, a middle dose of this precursor peptide $(0.3 \mathrm{nM})$ has a significant inhibitory action on NE overflow in ischemic hearts. This action was markedly attenuated by treatment with SM19712 or A-192621, suggesting that exogenously applied big ET-1 is converted to ET-1 by ECE, locally in the heart, and this ET-1 binds to $\mathrm{ET}_{\mathrm{B}} \mathrm{Rs}$ to exert its related beneficial action. However, it is important to note that the dose-response curve of the exogenous big ET-1-induced depressive effect on NE overflow was bellshaped $(0.3>1>0.1 \mathrm{nM})$. One of the possible reasons is that $\mathrm{ET}_{\mathrm{B}} \mathrm{Rs}$ may already be saturated by treatment with a middle dose of big ET-1, considering the result that combination of exogenous big ET-1 (0.3 nM) and ABT-627 did not produce a synergetic effect on NE overflow. Briefly, it has been proposed that a certain amount of ET-1 generated from exogenously applied big ET-1 preferentially acts on $\mathrm{ET}_{\mathrm{B}} \mathrm{Rs}$, but excessively generated ET-1 acts also on $\mathrm{ET}_{\mathrm{A}} \mathrm{Rs}$ in ischemic hearts. We hypothesize functional coupling of $\mathrm{ET}_{\mathrm{B}} \mathrm{Rs}$ and ECE-1, a dominant subtype of $\mathrm{ECE}$, as for the reason why this phenomenon occurs. ECE1 is classified into four isoforms based on differences in subcellular distribution [44]. Emoto et al. described that the isoform cleaving big ET-1 is different between endogenously generated and exogenously applied ET-1; the former is mainly catalyzed by isoforms expressed intracellularly, whereas the latter is cleaved by those expressed on the cell surface [45]. Based on this paper, endogenous ET-1 should be mostly generated inside the cell, whereas exogenously applied big ET-1 may be cleaved at the cell surface. In short, this isoform expressed on the cell surface would be positionally and/or functionally coupled to $\mathrm{ET}_{\mathrm{B}} \mathrm{Rs}$. This is just a hypothesis and must be further investigated.

As mentioned above, although $\mathrm{ET}_{\mathrm{B}}$ Rs have been demonstrated to exist in cardiac sympathetic nerves [24], it is unclear whether the effect seen at the middle dose $(0.3 \mathrm{nM})$ of exogenous big ET-1 is through the activation of $\mathrm{ET}_{\mathrm{B}} \mathrm{Rs}$ existing at this site. However, this may not be the case because the exogenous big ET-1-induced inhibitory effect on NE overflow after 40-min global ischemia/reperfusion was completely cancelled by treatment with the nitric oxide (NO) synthase (NOS) inhibitor $\mathrm{N}^{\mathrm{G}}$-nitro-L-arginine (NOARG) [46]. This finding suggests the involvement of $\mathrm{NO}$ derived from NOS in $\mathrm{ET}_{\mathrm{B}} \mathrm{R}$-mediated inhibitory modulation of carrier-mediated NE release. Although there are three different isoforms of NOS, neuronal NOS (NOS1), inducible NOS (NOS2), and endothelial NOS (NOS3), none have been confirmed to be normally distributed in sympathetic nerves [47, 48]. Therefore, ET-1 generated from exogenously applied big ET-1 is expected to bind to $\mathrm{ET}_{\mathrm{B}}$ Rs existing in cells other than sympathetic nerves, for instance endothelium, cardiomyocyte, or nitrergic nerves. Further studies are required to clarify the role of $\mathrm{ET}_{\mathrm{B}} \mathrm{Rs}$ existing in cardiac sympathetic nerves in carrier-mediated NE release.

There are a number of possible mechanisms for negative modulation of carrier-mediated NE release by NO. As noted above, carrier-mediated NE release from cardiac sympathetic nerve endings is triggered by inhibition of ATPase activity due to ATP depletion and activation of NHE [12]. In this regard, NO has been demonstrated to improve $\mathrm{Na}^{+} / \mathrm{K}^{+}$ ATPase function [49] or inhibit NHE function [50, 51] under cardiac pathological conditions. On the other hand, in culture experiments using PC-12 cells, another investigation has shown that NO negatively modifies NET activity in sympathetic neurons by nitrosation of regulatory sites on the transporter [52]. Again, further research is required to identify how NO suppresses carrier-mediated NE release from cardiac sympathetic nerve endings in protracted myocardial ischemia.

The inhibitory effect of exogenous big ET-1 on NE overflow $(0.3>1>0.1 \mathrm{nM})$ correlated well with the recovery of left ventricular systolic and diastolic function after ischemia/reperfusion in isolated perfused rat hearts [41]. Briefly, big ET-1 at a concentration of $0.3 \mathrm{nM}$ most efficiently improved left ventricular dysfunction after reperfusion. In addition, this beneficial effect of exogenously applied big ET1 was canceled by respective treatment with SM-19712, A192621, and NOARG $[41,46]$.

\section{Clinical Implications}

It is impossible to directly apply the above findings to a clinical setting mainly because of ex vivo data. However, previous in vivo studies of myocardial ischemia in animal models have provided clinically relevant information [25-29, 53]. In accordance with ex vivo data, each of the selective $\mathrm{ET}_{\mathrm{A}} \mathrm{R}$ antagonists $[26,28]$, the nonselective $\mathrm{ET}_{\mathrm{A}} \mathrm{R} / \mathrm{ET}_{\mathrm{B}} \mathrm{R}$ antagonist $[25,27,28]$ and the ECE inhibitor [29] have been reported to suppress increases in NE levels resulting from myocardial ischemia. For example, Fraccarollo et al. [26] demonstrated that long-term treatment with the selective $\mathrm{ET}_{\mathrm{A}} \mathrm{R}$ antagonist LU135252 decreased plasma NE concentrations 12 weeks after proximal left coronary artery ligation in rats. Lee et al. [28] showed the inhibitory effect of the selective $\mathrm{ET}_{\mathrm{A}} \mathrm{R}$ antagonist $\mathrm{ABT}-627$ or nonselective $\mathrm{ET}_{\mathrm{A}} \mathrm{R} / \mathrm{ET}_{\mathrm{B}} \mathrm{R}$ antagonist bosentan on regional myocardial interstitial and left ventricle NE levels 4 weeks after myocardial infarction induced by ligation of the anterior descending artery in rats. In this study, these ETR antagonists improved left ventricular systolic and diastolic function in postinfarcted rats. Also, in a study by Mulder and associates [25], bosentan decreased plasma NE levels in rats subjected to permanent left coronary artery ligation for 2 or 9 months. Likewise, Kolettis et al. [27] reported that bosentan markedly decreased serum NE levels $24 \mathrm{~h}$ postligation of the left coronary artery as well as the total duration of ventricular tachyarrhythmias during the delayed phase $(1-24 \mathrm{~h})$ postligation in rats. In addition, an orally active ECE inhibitor PP36 has been indicated to suppress cardiac tissue NE release $48 \mathrm{~h}$ after acute myocardial ischemia due to microsphere embolization of coronary 


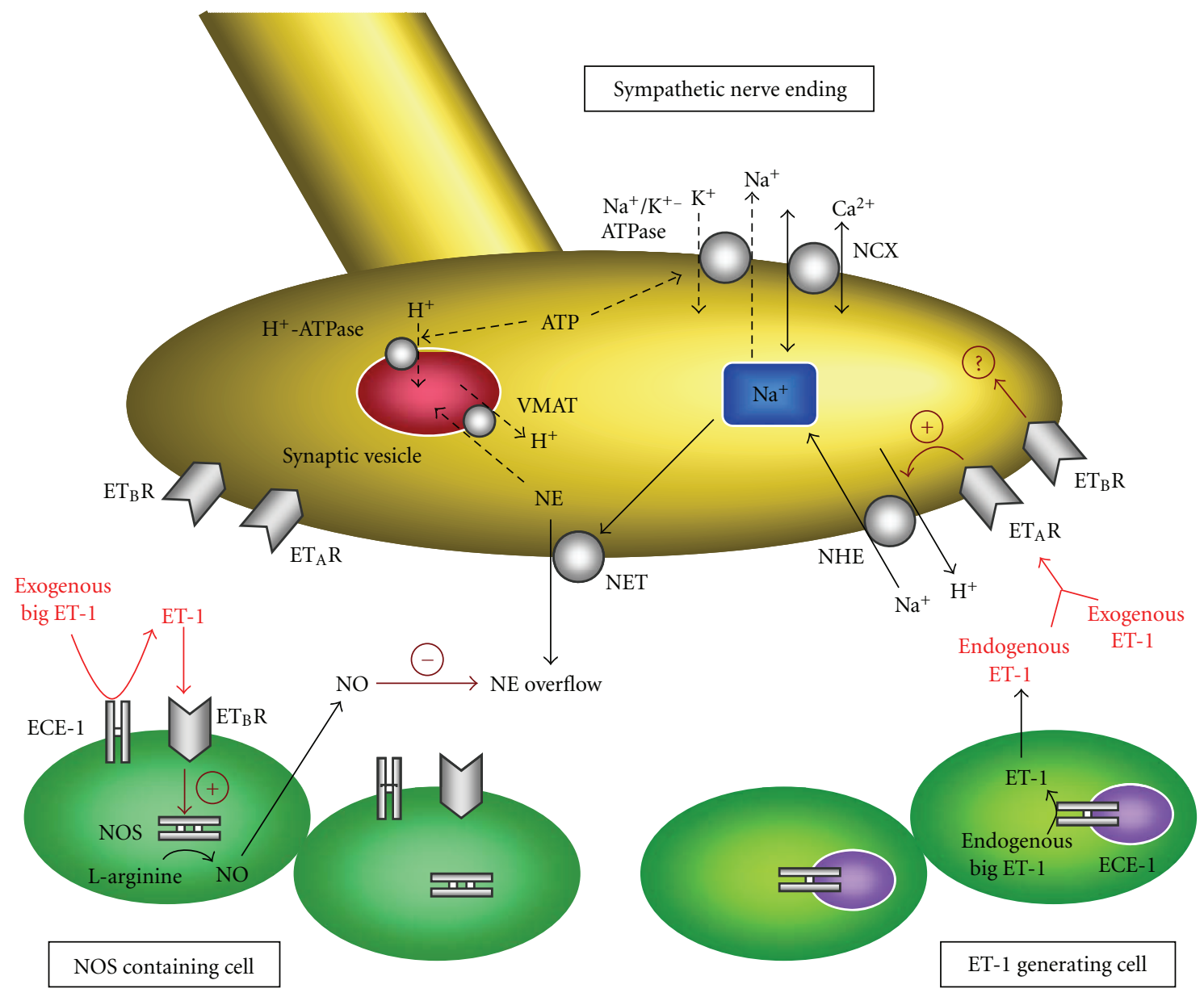

FIGURE 1: Summarizing scheme illustrating the interaction between the ET-1 system and carrier-mediated NE release in protracted myocardial ischemia. Stimulation of $\mathrm{ET}_{\mathrm{A}} \mathrm{Rs}$ existing in sympathetic nerve endings by endogenously generated or exogenously applied ET-1 increases neuronal NHE activity, thus potentiating carrier-mediated NE release. In contrast, exogenously applied big ET-1 is converted to ET1 by cell surface ECE-1, and this ET-1 preferentially binds to $\mathrm{ET}_{\mathrm{B}}$ Rs located on NOS containing cells to produce NO. Endogenously generated NO works to prevent carrier-mediated NE release. NCX, $\mathrm{Na}^{+} / \mathrm{Ca}^{2+}$ exchanger; VMAT: vesicular monoamine transporter.

microcirculation in rats [29]. Left ventricular contractility was partially restored and distention significantly improved by PP36 treatment in this research. Furthermore, an in vivo study using a genetic model was also consistent with the results of an ex vivo study. Oikonomidis et al. [53] recently demonstrated that serum NE levels during the early phase of myocardial infarction induced by left coronary artery ligation were much higher in $\mathrm{ET}_{\mathrm{B}} \mathrm{R}$-deficient $s l / s l$ rats than in wild-type rats. In this study, the severity of ventricular tachyarrhythmias during the 1st h postmyocardial infarction was also greater in $\mathrm{ET}_{\mathrm{B}} \mathrm{R}$-deficient rats, indicating that $\mathrm{ET}_{\mathrm{B}} \mathrm{R}$ decreases sympathetic activation and arrhythmogenesis during the early phase of myocardial infarction. Taken together, $\mathrm{ET}_{\mathrm{A}} \mathrm{R}$ antagonists or ECE inhibitors can be expected to suppress sympathetic overactivity with excessive NE release and following cardiac dysfunction in patients with ischemic cardiac disease. As a matter of course, however, we must keep in mind that NE overflow is only one of the risk factors for the development of cardiac injury, and also that ET-1 possesses many other pathophysiological actions. In other words, although this paper focused on functional myocardial damage caused by ischemia, we should not forget that morphological alterations such as infarction, hypertrophy, apoptosis, or fibrosis are also contributive to myocardial ischemia/reperfusion injury.

\section{Conclusion}

In summary (see Figure 1), during protracted myocardial ischemia, endogenously generated or exogenously applied ET-1 activates $\mathrm{ET}_{\mathrm{A}} \mathrm{Rs}$ in sympathetic nerve endings and stimulates neuronal NHE. As a result of NHE stimulation, $\mathrm{NE}$ is excessively released by potentiating carrier-mediated efflux. In contrast, ET-1 produced by appropriate amounts of exogenous big ET-1 works to prevent NE overflow by preferentially binding to $\mathrm{ET}_{\mathrm{B}}$ Rs located on NOS containing cells and producing NO. On the other hand, the role of $\mathrm{ET}_{\mathrm{B}} \mathrm{R}$ at cardiac sympathetic nerves in carrier-mediated $\mathrm{NE}$ release remains to be determined. Finally, a better understanding of the ET-1 system in NE overflow induced by 
protracted myocardial ischemia can lead to better treatments for ischemic cardiovascular diseases.

\section{Acknowledgment}

The authors thank Daniel Mrozek for reading the paper.

\section{References}

[1] G. Heusch, " $\alpha$-Adrenergic mechanisms in myocardial ischemia," Circulation, vol. 81, no. 1, pp. 1-13, 1990.

[2] C. J. H. Jones, L. Kuo, M. J. Davis, and W. M. Chilian, “ $\alpha$ adrenergic responses of isolated canine coronary microvessels," Basic Research in Cardiology, vol. 90, no. 1, pp. 61-69, 1995.

[3] G. Simonis, R. Marquetant, J. Röthele, and R. H. Strasser, “The cardiac adrenergic system in ischaemia: differential role of acidosis and energy depletion," Cardiovascular Research, vol. 38, no. 3, pp. 646-654, 1998.

[4] N. Dzimiri, "Regulation of $\beta$-adrenoceptor signaling in cardiac function and disease," Pharmacological Reviews, vol. 51, no. 3, pp. 465-501, 1999.

[5] G. Heusch, D. Baumgart, P. Camici et al., " $\alpha$-Adrenergic coronary vasoconstriction and myocardial ischemia in humans," Circulation, vol. 101, no. 6, pp. 689-694, 2000.

[6] K. Leineweber, G. Heusch, and R. Schulz, "Regulation and role of the presynaptic and myocardial $\mathrm{Na}^{+} / \mathrm{H}^{+}$exchanger NHE1: effects on the sympathetic nervous system in heart failure," Cardiovascular Drug Reviews, vol. 25, no. 2, pp. 123-131, 2007.

[7] A. Schömig, M. Haass, and G. Richardt, "Catecholamine release and arrhythmias in acute myocardial ischaemia," European Heart Journal, vol. 12, pp. 38-47, 1991.

[8] A. P. Waldenström, A. C. Hjalmarson, and L. Thornell, "A possible role of noradrenaline in the development of myocardial infarction. An experimental study in the isolated rat heart," American Heart Journal, vol. 95, no. 1, pp. 43-51, 1978.

[9] M. Imamura, H. M. Lander, and R. Levi, "Activation of histamine H3-receptors inhibits carrier-mediated norepinephrine release during protracted myocardial ischemia: comparison with adenosine A1-receptors and $\alpha 2$-adrenoceptors," Circulation Research, vol. 78, no. 3, pp. 475-481, 1996.

[10] E. Hatta, R. Maruyama, S. J. Marshall, M. Imamura, and R. Levi, "Bradykinin promotes ischemic norepinephrine release in guinea pig and human hearts," Journal of Pharmacology and Experimental Therapeutics, vol. 288, no. 3, pp. 919-927, 1999.

[11] R. Levi, R. B. Silver, C. J. Mackins, N. Seyedi, and M. Koyama, "Activation of a renin-angiotensin system in ischemic cardiac sympathetic nerve endings and its association with norepinephrine release," International Immunopharmacology, vol. 2, no. 13-14, pp. 1965-1973, 2002.

[12] A. Schömig, T. Kurz, G. Richardt, and E. Schömig, "Neuronal sodium homoeostasis and axoplasmic amine concentration determine calcium-independent noradrenaline release in normoxic and ischemic rat heart," Circulation Research, vol. 63, no. 1, pp. 214-226, 1988.

[13] M. Yanagisawa, H. Kurihara, S. Kimura et al., "A novel potent vasoconstrictor peptide produced by vascular endothelial cells," Nature, vol. 332, no. 6163, pp. 411-415, 1988.

[14] W. G. Haynes and D. J. Webb, "Endothelin as a regulator of cardiovascular function in health and disease," Journal of Hypertension, vol. 16, no. 8, pp. 1081-1098, 1998.

[15] K. Matsuyama, H. Yasue, K. Okumura et al., "Increased plasma level of endothelin-1-like immunoreactivity during coronary spasm in patients with coronary spastic angina," American Journal of Cardiology, vol. 68, no. 10, pp. 991-995, 1991.

[16] T. Miyauchi, M. Yanagisawa, T. Tomizawa et al., "Increased plasma concentrations of endothelin-1 and big endothelin-1 in acute myocardial infarction," The Lancet, vol. 2, no. 8653, pp. 53-54, 1989.

[17] F. Brunner, E. F. Du Toit, and L. H. Opie, "Endothelin release during ischaemia and reperfusion of isolated perfused rat hearts," Journal of Molecular and Cellular Cardiology, vol. 24, no. 11, pp. 1291-1305, 1992.

[18] M. Tawa, T. Fukumoto, M. Ohkita, and Y. Matsumura, "Role of endogenous endothelin-1 in post-ischemic cardiac dysfunction and norepinephrine overflow in rat hearts," European Journal of Pharmacology, vol. 591, no. 1-3, pp. 182-188, 2008.

[19] T. Watanabe, N. Suzuki, N. Shimamoto, M. Fujino, and A. Imada, "Contribution of endogenous endothelin to the extension of myocardial infarct size in rats," Circulation Research, vol. 69, no. 2, pp. 370-377, 1991.

[20] G. G. Serneri, I. Cecioni, S. Vanni et al., "Selective upregulation of cardiac endothelin system in patients with ischemic but not idiopathic dilated cardiomyopathy: endothelin-1 system in the human failing heart," Circulation Research, vol. 86, no. 4, pp. 377-385, 2000.

[21] X. S. Li, Q. D. Wang, and J. Pernow, "Beneficial effects of the endothelin receptor antagonist bosentan on myocardial and endothelial injury following ischaemia/reperfusion in the rat," European Journal of Pharmacology, vol. 283, no. 1-3, pp. 161168, 1995.

[22] B. Illing, M. Horn, H. Han et al., "Protective effect of the specific endothelin-1 antagonist BQ610 on mechanical function and energy metabolism during ischemia/reperfusion injury in isolated perfused rat hearts," Journal of Cardiovascular Pharmacology, vol. 27, no. 4, pp. 487-494, 1996.

[23] S. Yamamoto, N. Matsumoto, M. Kanazawa et al., "Different contributions of endothelin-A and endothelin-B receptors in postischemic cardiac dysfunction and norepinephrine overflow in rat hearts," Circulation, vol. 111, no. 3, pp. 302309, 2005.

[24] M. Isaka, A. Kudo, M. Imamura, H. Kawakami, and K. Yasuda, "Endothelin receptors, localized in sympathetic nerve terminals of the heart, modulate norepinephrine release and reperfusion arrhythmias," Basic Research in Cardiology, vol. 102, no. 2, pp. 154-162, 2007.

[25] P. Mulder, V. Richard, G. Derumeaux et al., "Role of endogenous endothelin in chronic heart failure: effect of long-term treatment with an endothelin antagonist on survival, hemodynamics, and cardiac remodeling," Circulation, vol. 96, no. 6, pp. 1976-1982, 1997.

[26] D. Fraccarollo, J. Bauersachs, M. Kellner, P. Galuppo, and G. Ertl, "Cardioprotection by long-term ETA receptor blockade and ACE inhibition in rats with congestive heart failure: mono-versus combination therapy," Cardiovascular Research, vol. 54, no. 1, pp. 85-94, 2002.

[27] T. M. Kolettis, G. G. Baltogiannis, D. G. Tsalikakis et al., "Effects of dual endothelin receptor blockade on sympathetic activation and arrhythmogenesis during acute myocardial infarction in rats," European Journal of Pharmacology, vol. 580, no. 1-2, pp. 241-249, 2008.

[28] T. M. Lee, C. C. Chen, M. S. Lin, and N. C. Chang, "Effect of endothelin receptor antagonists on ventricular susceptibility in postinfarcted rats," American Journal of Physiology, vol. 294, no. 4, pp. H1871-H1879, 2008.

[29] V. A. Rufanova, V. F. Pozdnev, E. I. Kalenikova et al., "Endothelin-converting enzyme inhibition in the rat model of acute 
heart failure: heart function and neurohormonal activation," Experimental Biology and Medicine, vol. 234, no. 10, pp. 12011211, 2009.

[30] Z. S. Kyriakides, D. T. Kremastinos, F. Kolokathis, A. Kostopoulou, M. Georgiadis, and D. J. Webb, "Acute endothelin A receptor antagonism improves coronary artery compliance in coronary artery disease patients," Clinical Science, vol. 103, supplement 48, pp. 179S-183S, 2002.

[31] C. M. O'Connor, W. A. Gattis, K. F. Adams et al., “Tezosentan in patients with acute heart failure and acute coronary syndromes: results of the randomized intravenous tezosentan study (RITZ-4)," Journal of the American College of Cardiology, vol. 41, no. 9, pp. 1452-1457, 2003.

[32] R. Lariviere, L. Y. Deng, R. Day, P. Sventek, G. Thibault, and E. L. Schiffrin, "Increased endothelin-1 gene expression in the endothelium of coronary arteries and endocardium in the DOCA-salt hypertensive rat," Journal of Molecular and Cellular Cardiology, vol. 27, no. 10, pp. 2123-2131, 1995.

[33] O. S. Opgaard, M. Adner, T. H. F. Peters et al., "Endocardial expression and functional characterization of endothelin-1," Molecular and Cellular Biochemistry, vol. 224, no. 1-2, pp. 151$158,2001$.

[34] D. Merkus, A. K. Brzezinska, C. Zhang, S. Saito, and W. M. Chilian, "Cardiac myocytes control release of endothelin-1 in coronary vasculature," American Journal of Physiology, vol. 288, no. 5, pp. H2088-H2092, 2005.

[35] G. M. Rubanyi and M. A. Polokoff, "Endothelins: molecular biology, biochemistry, pharmacology, physiology, and pathophysiology," Pharmacological Reviews, vol. 46, no. 3, pp. 325415, 1994.

[36] C. R. Bacon and A. P. Davenport, "Endothelin receptors in human coronary artery and aorta," British Journal of Pharmacology, vol. 117, no. 5, pp. 986-992, 1996.

[37] P. Molenaar, G. O'Reilly, A. Sharkey et al., "Characterization and localization of endothelin receptor subtypes in the human atrioventricular conducting system and myocardium," Circulation Research, vol. 72, no. 3, pp. 526-538, 1993.

[38] M. Avkiran and R. S. Haworth, "Regulatory effects of G protein-coupled receptors on cardiac sarcolemmal $\mathrm{Na}^{+} / \mathrm{H}^{+}$ exchanger activity: signalling and significance," Cardiovascular Research, vol. 57, no. 4, pp. 942-952, 2003.

[39] R. A. Dulce, C. Hurtado, I. L. Ennis et al., "Endothelin-1 induced hypertrophic effect in neonatal rat cardiomyocytes: involvement of $\mathrm{Na}^{+} / \mathrm{H}^{+}$and $\mathrm{Na}^{+} / \mathrm{Ca}^{+}$exchangers," Journal of Molecular and Cellular Cardiology, vol. 41, no. 5, pp. 807-815, 2006.

[40] T. Horinouchi, H. Asano, T. Higa et al., "Differential coupling of human endothelin type A receptor to G q/11 and G12 proteins: the functional significance of receptor expression level in generating multiple receptor signaling," Journal of Pharmacological Sciences, vol. 111, no. 4, pp. 338-351, 2009.

[41] M. Tawa, T. Fukumoto, M. Ohkita et al., "Effects of exogenous big endothelin-1 on postischemic cardiac dysfunction and norepinephrine overflow in rat hearts," Hypertension Research, vol. 34, no. 2, pp. 218-224, 2011.

[42] S. Kimura, Y. Kasuya, T. Sawamura et al., "Conversion of big endothelin-1 to 21-residue endothelin-1 is essential for expression of full vasoconstrictor activity: structure-activity relationships of big endothelin-1," Journal of Cardiovascular Pharmacology, vol. 13, supplement 5, pp. S5-S7, 1989.

[43] C. Parnot, J. M. Le Moullec, M. A. Cousin, D. Guédin, P. Corvol, and F. Pinet, "A live-cell assay for studying extracellular and intracellular endothelin-converting enzyme activity," Hypertension, vol. 30, no. 4, pp. 837-844, 1997.
[44] O. Valdenaire, D. Lepailleur-Enouf, G. Egidy et al., "A fourth isoform of endothelin-converting enzyme (ECE-1) is generated from an additional promoter: molecular cloning and characterization," European Journal of Biochemistry, vol. 264, no. 2, pp. 341-349, 1999.

[45] N. Emoto, Y. Nurhantari, H. Alimsardjono et al., "Constitutive lysosomal targeting and degradation of bovine endothelinconverting enzyme-1a mediated by novel signals in its alternatively spliced cytoplasmic tail," The Journal of Biological Chemistry, vol. 274, no. 3, pp. 1509-1518, 1999.

[46] M. Tawa, T. Fukumoto, M. Ohkita et al., "Contribution of nitric oxide in big endothelin-1-induced cardioprotective effects on ischemia/reperfusion injury in rat hearts," Journal of Cardiovascular Pharmacology, vol. 57, no. 5, pp. 575-578, 2011.

[47] L. Klimaschewski, W. Kummer, B. Mayer et al., "Nitric oxide synthase in cardiac nerve fibers and neurons of rat and guinea pig heart," Circulation Research, vol. 71, no. 6, pp. 1533-1537, 1992.

[48] P. Alm, B. Uvelius, J. Ekstrom, B. Holmqvist, B. Larsson, and K. E. Andersson, "Nitric oxide synthase-containing neurons in rat parasympathetic, sympathetic and sensory ganglia: a comparative study," Histochemical Journal, vol. 27, no. 10, pp. 819831, 1995.

[49] K. Y. Xu, S. P. Kuppusamy, J. Q. Wang et al., "Nitric oxide protects cardiac sarcolemmal membrane enzyme function and ion active transport against ischemia-induced inactivation," The Journal of Biological Chemistry, vol. 278, no. 43, pp. 41798-41803, 2003.

[50] N. Ito, J. Bartunek, K. W. Spitzer, and B. H. Lorell, "Effects of the nitric oxide donor sodium nitroprusside on intracellular $\mathrm{pH}$ and contraction in hypertrophied myocytes," Circulation, vol. 95, no. 9, pp. 2303-2311, 1997.

[51] S. E. Anderson, D. M. Kirkland, A. Beyschau, and P. M. Cala, "Acute effects of $17 \beta$-estradiol on myocardial $\mathrm{pH}, \mathrm{Na}^{+}$, and $\mathrm{Ca}^{+}$and ischemia-reperfusion injury," American Journal of Physiology, vol. 288, no. 1, pp. C57-C64, 2005.

[52] D. M. Kaye, S. D. Wiviott, L. Kobzik, R. A. Kelly, and T. W. Smith, "S-nitrosothiols inhibit neuronal norepinephrine transport," American Journal of Physiology, vol. 272, no. 2, pp. H875-H883, 1997.

[53] D. L. Oikonomidis, D. G. Tsalikakis, G. G. Baltogiannis et al., "Endothelin-B receptors and ventricular arrhythmogenesis in the rat model of acute myocardial infarction," Basic Research in Cardiology, vol. 105, no. 2, pp. 235-245, 2010. 


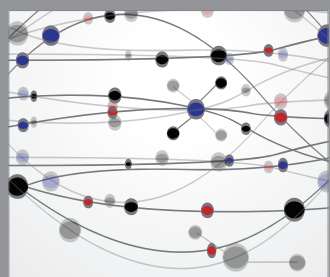

The Scientific World Journal
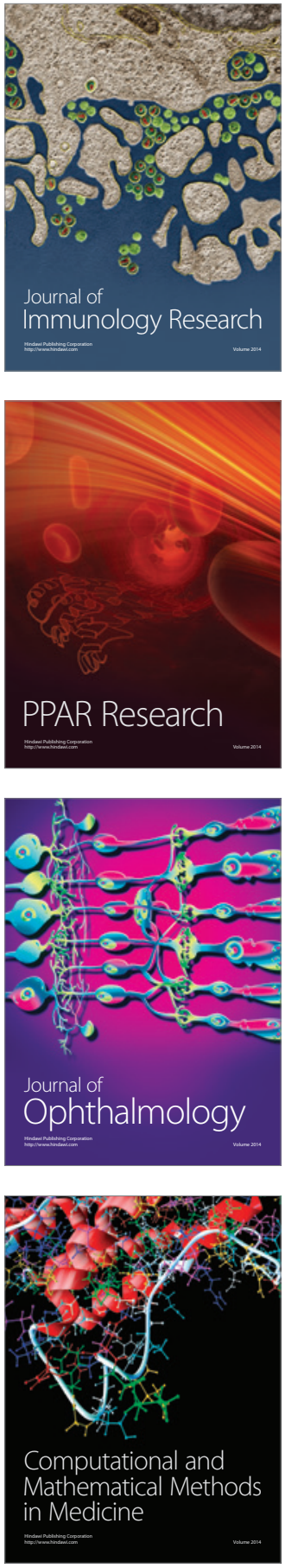

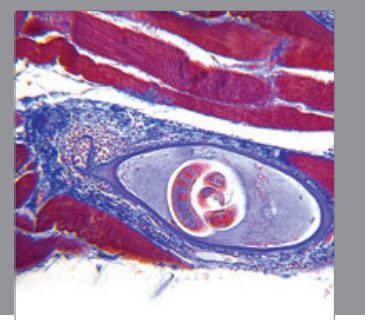

Gastroenterology

Research and Practice
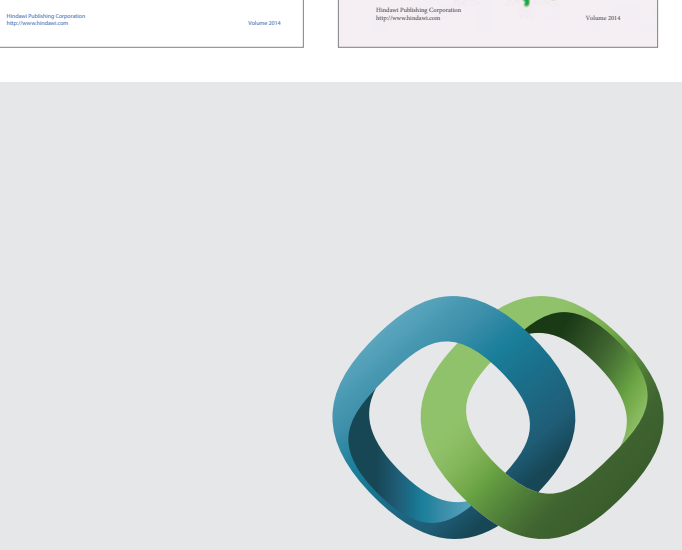

\section{Hindawi}

Submit your manuscripts at

http://www.hindawi.com
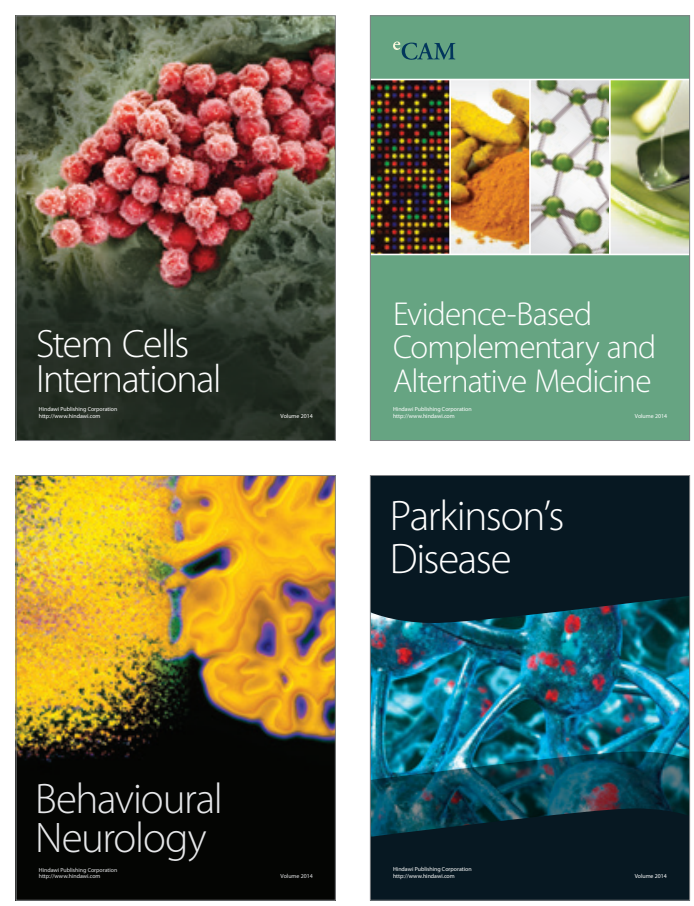

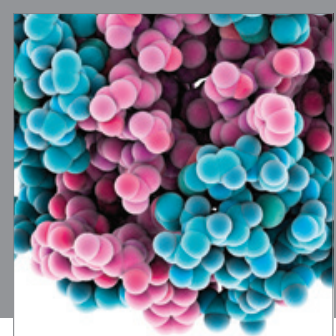

Journal of
Diabetes Research

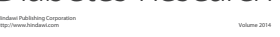

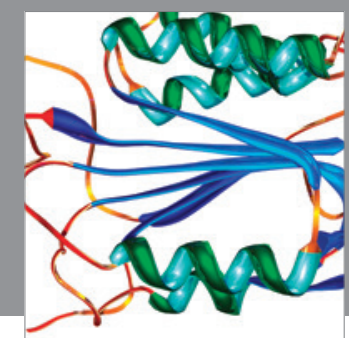

Disease Markers
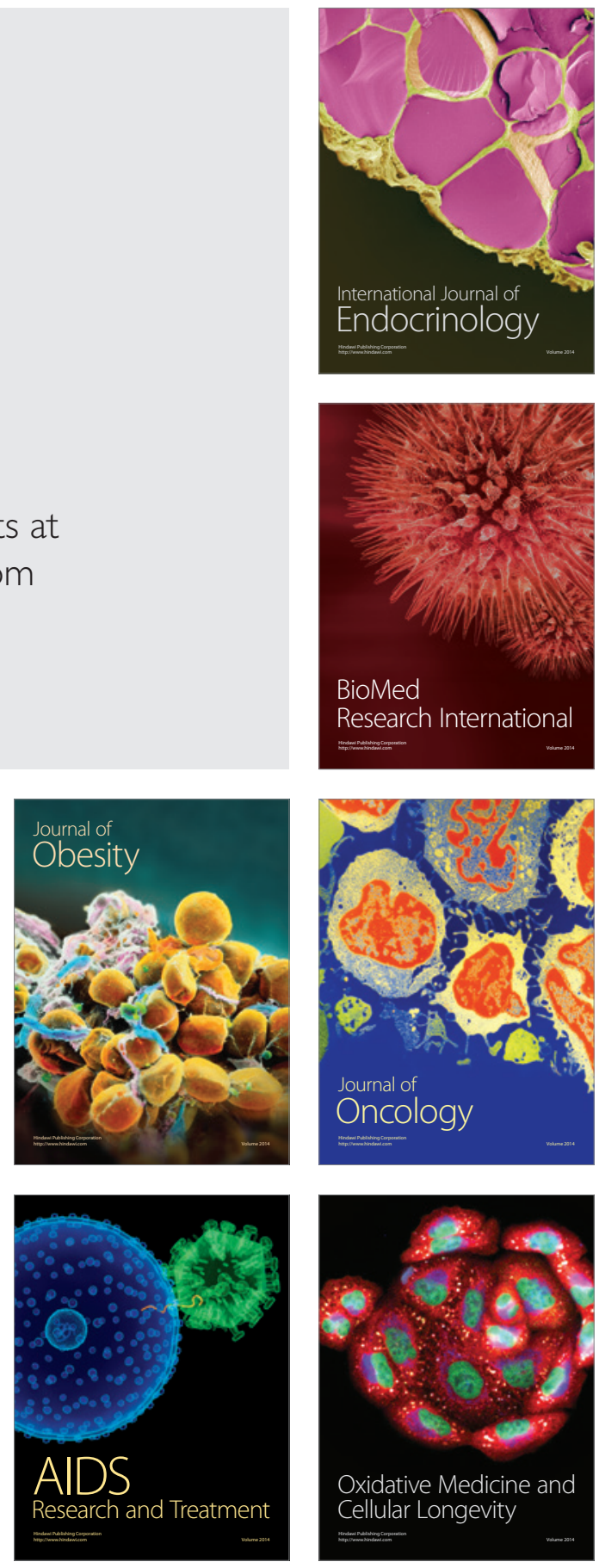